\title{
How phosphoubiquitin activates Parkin
}

\author{
Cell Research (2015) 25:1087-1088. doi:10.1038/cr.2015.97; published online 11 August 2015
}

\section{A recent report, solving the struc- ture of a Parkin-phosphoubiquitin complex, greatly advances the un- derstanding of the Parkin activation mechanism.}

Pink1, a mitochondrial protein kinase, and Parkin, a cytosolic E3 ubiquitin ligase, mutated in hereditary forms of early onset Parkinson's disease (PD), function together in mitochondrial maintenance. Pink1 is imported into healthy mitochondria with normal membrane potential, and then cleaved by the PARL protease and eventually degraded in the proteasome. When mitochondria lose their membrane potential, Pink1 remains exposed on the mitochondrial outer surface, recruiting Parkin and activating its E3 activity to trigger extensive mitochondrial protein ubiquitination and subsequent clearance of the damaged mitochondria through autophagy [1].

Parkin belongs to the RBR (RINGin-Between-RING) E3 ligase family, which has two tandem RING domains — the RING1 domain binds ubiquitincharged E2 enzymes and transfers the ubiquitin to a catalytic cysteine residue in the RING2 domain before conjugating it to a substrate. Parkin E3 activity is autoinhibited through multiple intramolecular interactions (Figure 1): E2 binding is blocked by the N-terminal ubiquitin-like (UBL) domain as well as a 'repressor' element (REP); the catalytic cysteine in the RING2 domain is masked by a unique Parkin domain (UPD, also known as RING0) [2, 3]. Parkin E3 activity is stimulated by Pink1 through two Ser65 phosphorylation events, one on the Parkin UBL domain and the other on the equivalent serine in ubiquitin [1]. UBL domain phosphorylation causes its dissociation from the RBR core; however, the mechanism through which phosphoubiquitin activates Parkin is unclear.

To understand how phosphoubiquitin induces Parkin activation, Wauer and colleagues obtained a crystal structure of truncated Parkin (140-461) in complex with phospho-Ser65 ubiquitin [4]. They used human body louse Parkin, which contains a cysteine near its ubiquitin-binding site that can react with a ubiquitin suicide probe to form a stable complex for purification. The structure reveals that phosphoubiquitin has extensive interactions with multiple domains of Parkin. Phosphoubiquitin interacts with the RING1 domain through its hydrophobic Ile44 patch, which is used for many Ub interactions; and its C-terminus also forms a close contact with the IBR domain (Figure 1).

The structure was further confirmed by a series of compelling functional analyses. Mutations in the predicted phosphate-binding pocket of Parkin, such as His302, greatly decreased phosphoubiquitin binding. Importantly, G284R, a PD patient mutation, was among them, thus supporting the physiological importance of phosphoubiquitin binding in Parkin function. Parkin E3 activity can be activated by phosphoubiquitin in autoubiquitination assays even in the absence of UBL domain phosphorylation. Several recent studies suggest that phosphoubiquitin binding is necessary for Parkin mitochondrial translocation [5-7]. Consistently, these phosphoubiquitin binding mutations not only impaired the phosphoubiquitininduced Parkin activation in autou- biquitination assays, but also crippled Parkin mitochondrial recruitment and localization.

Phosphoubiquitin binding induces profound structural changes that could explain Parkin activation. Phosphoubiquitin is bound to a straight helix in RING1; in contrast, this helix is kinked in the inactive Parkin. The straightening of this helix may trigger the rotation and movement of the IBR domain, thus stretching the IBR-REP linker, which in turn affects the binding interface between RBR and the UBL domain. Indeed, the authors showed that phosphoubiquitin can compete with and displace the UBL domain from the Parkin RBR core. Moreover, phosphoubiquitin binding appears to stimulate Parkin Ser65 phosphorylation by Pink1, as reported by Kazlauskaite et al. [8]. Using nuclear magnetic resonance, Wauer and colleagues showed that UBL domain phosphorylation disrupts its Ile44 patch, a binding interface between UBL and RBR core, further ensuring the dissociation of UBL domain and also explaining why pS65 UBL cannot bind to the pS65 Ub-binding pocket.

Do we completely understand Parkin activation? A puzzling observation is that ubiquitin-vinyl sulfone, which can conjugate to the catalytic cysteine when it is accessible, reacted with phosphoUBL Parkin but not phosphoubiquitinbound Parkin $[4,5]$, indicating that the catalytic cysteine may not be completely exposed in the latter case. One might ask whether the autoubiquitination of phosphoubiquitin-activated Parkin occurs through the catalytic cysteine, or alternatively is due to RING1 activity alone. Is UBL phosphorylation essen- 


\section{Parkin

Ubl UPD RING1 - IBR - REP - RING2
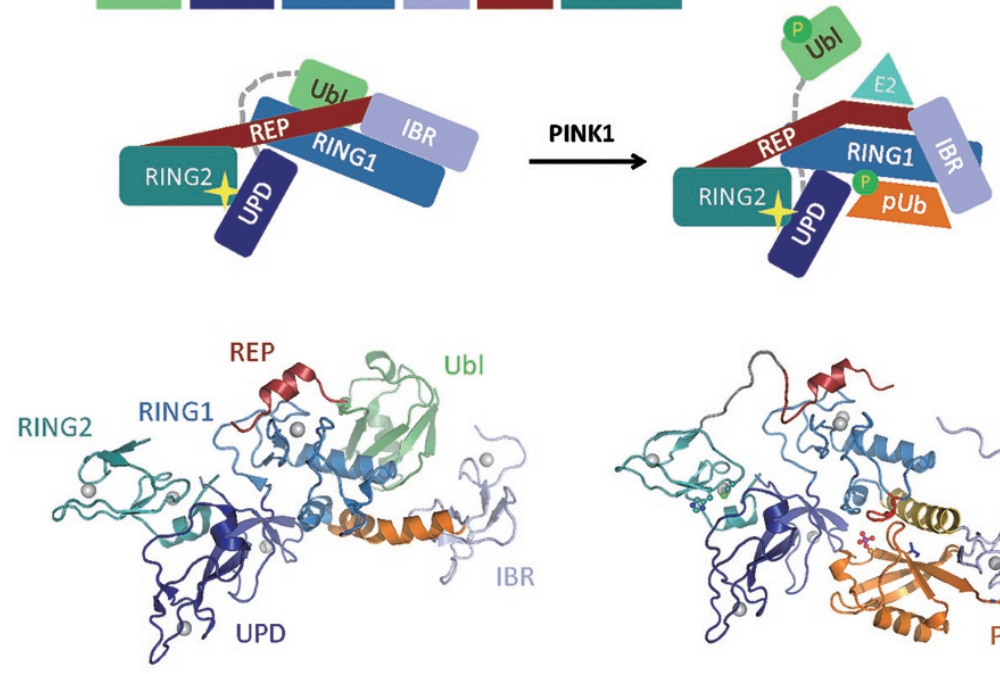

Full length Parkin

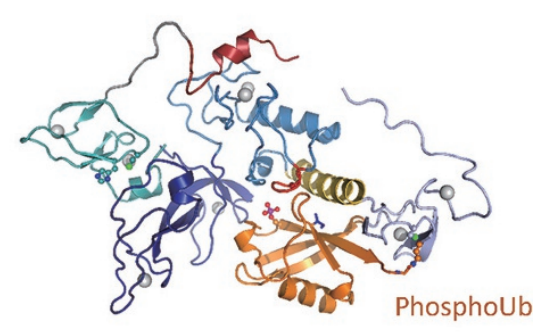

Parkin (140-461 aa) in complex with Ser65-phosphoubiquitin

Figure $1 \mathrm{~A}$ schematic domain map of Parkin and the complex of Parkin-phosphoUb. The yellow star mark represents the catalytic cysteine in the RING2 domain. The design is adapted from Wauer et al. [4], and the Parkin structural photos are kindly provided by Dr David Komander.

tial for exposing the catalytic cysteine? UBL domain deletion greatly diminishes ubiquitin trapping by Parkin C431S during mitophagy, indicating a positive role of UBL domain in Parkin activity [9]. In the current work, the authors proposed an intriguing hypothesis: the phosphoUBL domain may instead bind to the UPD through a second, distinct putative phosphate-binding pocket [3]. Consistent with this, they showed that mutations of this pocket blocked the E3 activity of phospho-UBL Parkin.

Parkin is reportedly capable of catalyzing multiple different ubiquitin linkage chain types, including K6, K11, $\mathrm{K} 48$, and $\mathrm{K} 63$ [5]. Interestingly, in cells expressing only S65A ubiquitin, the abundance of K6, K11, and K48 chains detected on mitochondria decrease dramatically [10]. Could Parkin have multiple different activation states: UBL phosphorylation alone, activation by phosphoubiquitin binding alone, or both? In this regard, it would be worth comparing the chain types formed by phospho-UBL Parkin and phosphoubiquitin-activated Parkin. Another issue is which E2 is used by Parkin during mitophagy. UBE2D and UBE2L3 have been shown to redundantly activate Parkin at the initial stage and be required for Parkin mitochondrial translocation, whereas UBE2N appears to act at a later stage in mitochondrial clustering $[11,12]$. It would be interesting to see whether Parkin associated with different E2 enzymes would catalyze different chain linkage types.

Because of its clinical importance and clearly-defined function in mitophagy, a key cellular quality control process, Parkin's biochemical mechanism is being rapidly unfolded. This will not only provide therapeutic opportunities, such as rescue of some Parkin mutants, but also generate valuable insights into this newly-identified RING-HECT hybrid E3 ligase family.

\section{Xinde Zheng ${ }^{1}$, Tony Hunter ${ }^{1}$}

${ }^{1}$ Molecular and Cell Biology Laboratory, Salk Institute for Biological Studies, 10010 North Torrey Pines Road, La Jolla, CA 92037, USA

Correspondence: Tony Hunter

Tel: 1+858-453-4100 ext 1385

E-mail: hunter@salk.edu

\section{References}

1 Pickrell AM, Youle RJ. Neuron 2015; 85:257-273.

2 Trempe JF, Sauvé V, Grenier K, et al. Science 2013; 340:1451-1455.

3 Wauer T, Komander D. EMBO J 2013; 32:2099-2112.

4 Wauer T, Simicek M, Schubert A, et al. Nature 2015; 524:370-374.

5 Ordureau A, Sarraf SA, Duda DM, et al. Mol Cell 2014; 56:360-375.

6 Shiba-Fukushima K, Arano T, Matsumoto $\mathrm{G}$, et al. PLoS Genet 2014; 10:e1004861.

7 Okatsu K, Koyano F, Kimura M, et al. J Cell Biol 2015; 209:111-128.

8 Kazlauskaite A, Martínez-Torres RJ, Wilkie S, et al. EMBO Rep 2015; pii: e201540352.

9 Zheng X, Hunter T. Cell Res 2013; 23:886897.

10 Ordureau A, Heo JM, Duda DM, et al. Proc Natl Acad Sci USA 2015; 112:6637-6642.

11 Fiesel FC, Moussaud-Lamodière EL, Ando M, et al. J Cell Sci 2014; 127:3488-3504.

12 Geisler S, Vollmer S, Golombek S, et al. $J$ Cell Sci 2014; 127:3280-3293. 